\title{
Determinação de uma fórmula para calcular os termos de uma sequência de Fibonacci por meio de progressões geométricas e espaços vetoriais
}

\section{Determination of a formula for calculating the terms of a Fibonacci sequence by means of geometric progressions and vector spaces}

\author{
Allan Kenedy Santos Silva \\ Universidade Federal de Alagoas (UFAL), Campus Sertão \\ Delmiro Gouveia, AL, Brasil \\ allankenedy82@gmail.com
}

\begin{tabular}{l}
\hline Informações do Artigo \\
\hline cc) (7) \$ \\
\hline $\mathrm{EY}$
\end{tabular}

Histórico do Artigo

Submissão: 15 de julho de 2018.

Aceite: 28 de setembro de 2018 .

\begin{abstract}
Resumo
A famosa sequência de Fibonacci surgiu de um simples problema de reprodução de coelhos. Devido a diversas aparições na natureza, aplicações na Matemática, Ciências, Arquitetura e Estética, juntamente com sua relação com o número de ouro, a sequência de Fibonacci tornou-se uma das mais importantes da Matemática. Neste artigo, as sequências de Fibonacci serão abordadas do ponto de vista da Álgebra Linear. Desse estudo será determinada uma fórmula fechada do termo geral da sequência. Será apresentada, também, a relação com o número de ouro. No final, será analisada uma versão simplificada da fórmula.
\end{abstract}

Palavras-chave

Sequência de Fibonacci

Número de Ouro

Progressão Geométrica

Álgebra Linear

Espaço Vetorial

\section{Keywords}

Fibonacci Sequence

Golden Number

Geometric Progression

Linear Algebra

Vector Space

\begin{abstract}
The famous Fibonacci sequence arose from a simple rabbit breeding problem. Due to various appearances in nature, applications in Mathematics, Science, Architecture and Aesthetics, along with its relation to the Golden number, the Fibonacci sequence has become one of the most important in Mathematics. In this paper, the Fibonacci sequences will be approached from the standpoint of Linear Algebra. From this study will be determined a closed formula of the general term of the sequence. The relationship with the golden number will also be shown. At the end a simplified version of the formula will be analyzed.
\end{abstract}

\section{Introdução}

Leonardo de Pisa nasceu em Pisa, na Toscana, Itália, por volta de 1175, e ficou conhecido como Leonardo Fibonacci, devido ao fato de Fibonacci ser uma composição por aglutinação de fillius Bonacci, que quer dizer filho de Bonacci - seu pai se chamava Guglielmo dei Bonnacci (RAMOS, 2013).

Por seu pai ser um importante mercador, Fibonacci viajou pelo Mediterrâneo por rotas comerciais já consolidadas, entrando em contato com países muçulmanos, assimilando conhecimento cultural e matemático desses países (RAMOS, 2013, FERREIRA, 2006).

Ao regressar a Pisa, Fibonacci passa a escrever trabalhos nos quais incorpora os conhecimentos que tinha adquirido com os árabes. O seu livro mais conhecido foi um tratado de aritmética e álgebra elementar intitulado Líber Abaci (Livro do Ábaco), escrito em 1202 (FERREIRA, 2006). 
No capítulo 12 do Líber Abaci, intitulado De solutionibus multarum positarum questionum quas erraticas appellamus (A solução de problemas diversos), apresenta-se um problema de reprodução de coelhos, que é o problema mais conhecido dentre todos os analisados por Fibonacci:

Um homem pôs um par de coelhos num lugar cercado por todos os lados por um muro. Quantos pares de coelhos podem ser gerados a partir desse par em um ano se, supostamente, todo mês cada par dá à luz um novo par, que é fértil a partir do segundo mês. (FERREIRA, 2006, p. 6).

Este problema é de solução simples:

- No $1^{\circ}$ mês, tem-se apenas um par de filhotes (1 par);

- No $2^{\circ}$ mês, o par de coelhos se torna adulto e, assim, fértil (1 par);

- No $3^{\circ}$ mês, o par de coelhos adultos gera um par de filhotes (2 pares);

- No $4^{\circ}$ mês o par de coelhos adultos gera um par de filhotes e os filhotes gerados no $3^{\circ}$ mês se tornam férteis (3 pares);

- No $5^{\circ}$ mês, os pares férteis do $1^{\circ}$ e $3^{\circ}$ mês geram um par de filhotes cada, e o par do $4^{\circ}$ mês se torna fértil (5 pares).

Continuando, obtém-se a seguinte sequência de pares de coelhos: $(1,1,2,3,5,7, \ldots)$. Esta é a famosa sequência de Fibonacci, a qual possui a propriedade de que um termo qualquer, a partir do $3^{\circ}$, é igual à soma dos dois termos antecedentes.

Ao longo dos anos, foram encontradas diversas propriedades envolvendo a sequência de Fibonacci, como por exemplo, o $n k$-ésimo termo da sequência supracitada é múltiplo do $k$-ésimo termo da mesma, para quaisquer naturais $n$ e $k$; a soma dos $n$ primeiros termos da sequência é uma unidade a menos que o termo de índice $n+2$, dentre outras propriedades (FERREIRA, 2006).

Outra importante propriedade é a que afirma que a razão entre termos sucessivos da sequência de Fibonacci converge para $\frac{1+\sqrt{5}}{2}$, o chamado número de ouro, à medida em que os índices dos termos crescem.

A sequência de Fibonacci possui várias aplicações. Em Ótica, é usada para calcular o número de reflexões de um raio de luz entre duas placas de vidro. Em Economia, é utilizada nos ciclos de Elliot (FERREIRA, 2006). Em Biologia, pode representar a população de abelhas em uma colmeia, a distribuição de sementes em girassóis, pétalas em crisântemos ou distribuição de folhas em plantas (SAMPAIO; SILVA, 2012, RAMOS, 2013). Devido às aplicações em Matemática, Ciências, Arquitetura e Estética, a sequência de Fibonacci e o número de ouro merecem, por si sós, um livro inteiro sobre eles. E esse livro existe! Foi escrito por H. E. Huntley (HUNTLEY, 1985).

Para calcular os termos de uma sequência de Fibonacci, é necessário determinar os termos anteriores, devido ao seu caráter recursivo. Por exemplo, para determinar o $500^{\circ}$ termo da sequência, é necessário conhecer os termos $499^{\circ}$ e $498^{\circ}$, e, para sabê-los, deve-se conhecer os anteriores e assim sucessivamente, até os primeiros termos. Nesse sentido, uma fórmula fechada 
(função apenas da posição e dos termos formadores da sequência) do termo geral se mostra bastante útil. Tal fórmula já existe, conhecida como fórmula de Binet, cuja dedução encontrada na literatura geralmente toma por base propriedades do número de ouro (FERREIRA, 2006, HUNTLEY, 1985), ou usa equações a diferenças finitas (SANTOS, 1998, OLIVEIRA, 2013, GUSMÃO, 2003). Contudo, este texto não tem o objetivo de determinar tal fórmula, mas sim mostrar uma abordagem alternativa de dedução usando Álgebra Linear. Uma abordagem similar foi feita por Ramos (2013), mas neste texto será apresentada de forma generalizada, isto é, para sequências que possuem a mesma lei de recursividade da de Fibonacci, cujos dois primeiros termos não são necessariamente iguais a 1, derivando assim a fórmula de Binet como um caso particular. Dessa fórmula será demonstrada a relação entre as sequências de Fibonacci e o número de ouro. Por fim, será feita a análise numérica de uma versão simplificada da fórmula, permitindo o cálculo aproximado dos termos da sequência.

\section{Espaço vetorial, base e dimensão}

Nesta seção, serão abordados, de forma breve, alguns conceitos básicos de Álgebra Linear, como espaço vetorial, dependência linear, base e dimensão. Se o leitor já possui familiaridade com tais conceitos, poderá, se preferir, omitir a leitura desta seção, podendo partir para a seção 3.

Definição 2.1: Um conjunto $V$ é um espaço vetorial real, ou simplesmente espaço vetorial, se nele estiverem definidas as operações: adição, que a cada par de vetores $u, v \in V$ associa ao vetor $u+v \in V$, e multiplicação, que a cada vetor $u \in V$ e a cada real $\alpha$ associa ao vetor $\alpha u \in V$, com as propriedades a seguir, $\forall u, v, w \in V$ e $\forall \alpha, \beta \in \mathbb{R}$ :

1. $u+v=v+u^{1}$

2. $u+(v+w)=(u+v)+w$;

3. Existe um vetor $0 \in V$ tal que $u+0=u$;

4. Existe um vetor $-u \in V$ tal que $u+(-u)=0$;

5. $\alpha(\beta u)=(\alpha \beta) u$;

6. $\alpha(u+v)=\alpha u+\alpha v$;

7. $(\alpha+\beta) u=\alpha u+\beta u$;

8. $1 \cdot u=u$;

Definição 2.2: Sejam $v_{1}, v_{2}, \ldots, v_{n}$ vetores do espaço vetorial $V$ e os escalares (números reais) $a_{1}, a_{2}, \ldots, a_{n}$. O vetor

$$
v=a_{1} v_{1}+a_{2} v_{2}+\cdots+a_{n} v_{n}
$$

é dito uma combinação linear dos vetores $v_{1}, v_{2}, \ldots, v_{n}$.

\footnotetext{
${ }^{1}$ Esta condição pode ser omitida como indicado por Lima (2016, p. 6, exercício 1.11).
} 
Definição 2.3: Os vetores $v_{1}, v_{2}, \ldots, v_{n}$ são ditos linearmente dependentes se existem escalares $a_{1}, a_{2}, \ldots, a_{n}$ não todos nulos tais que

$$
a_{1} v_{1}+a_{2} v_{2}+\cdots+a_{n} v_{n}=0,
$$

do contrário, $v_{1}, v_{2}, \ldots, v_{n}$ são ditos linearmente independentes.

Definição 2.4: Um subconjunto $E$ do espaço vetorial $V$ é um subespaço vetorial se, com as mesmas operações definidas no espaço $V, E$ tiver a estrutura de espaço vetorial. Em outros termos, $E$ é um subespaço de $V$ se $E \subseteq V$ e

1. $u, v \in E \Rightarrow u+v \in E$;

2. $\alpha \in \mathbb{R}, u \in E \Rightarrow \alpha u \in E$.

Definição 2.5: Sejam $V$ um espaço vetorial e $A=\left\{v_{1}, v_{2}, \ldots, v_{n}\right\} \subset V, A \neq \emptyset$. O subespaço vetorial gerado por $A$ é o conjunto

$$
G(A)=\left\{v=a_{1} v_{1}+a_{2} v_{2}+\cdots+a_{n} v_{n} ; a_{1}, a_{2}, \ldots, a_{n} \in \mathbb{R}\right\}
$$

isto é, é o conjunto das combinações lineares dos elementos de $A$.

Definição 2.6: Um conjunto $B=\left\{v_{1}, v_{2}, \ldots, v_{n}\right\} \subseteq V$ é uma base do espaço vetorial $V$ se:

1. $B$ é linearmente independente;

2. $B$ gera $V$, isto é, $V=G(B)$.

O número $n$ é a dimensão do espaço $\mathrm{V}$ e escreve-se $\mathrm{n}=\operatorname{dim} \mathrm{V}$.

O conceito de base é importantíssimo para a álgebra linear, pois qualquer vetor de um espaço $V$ pode ser escrito em termos de vetores básicos, e tal representação, uma vez fixada a base, é única, conforme apresentado em Steinbruch e Winterle (1997, p. 32). Uma base de um espaço vetorial pode ser encarado como a referência de um sistema de coordenadas. Assim, um vetor $v=a_{1} v_{1}+a_{2} v_{2}+\cdots+a_{n} v_{n} \in V$ pode ser escrito como $v=\left(a_{1}, a_{2}, \ldots, a_{n}\right)$ na base $B=$ $\left\{v_{1}, v_{2}, \ldots, v_{n}\right\}$, sendo univocamente determinado por meio das coordenadas $a_{1}, a_{2}, \ldots, a_{n}$.

Nesse contexto, dados $u=\left(a_{1}, a_{2}, \ldots, a_{n}\right)$ e $v=\left(b_{1}, b_{2}, \ldots, b_{n}\right)$ vetores em $V$ na base $B$, as operações de adição e multiplicação passam a ser definidas por

$$
\begin{gathered}
u+v=\left(a_{1}+b_{1}, a_{2}+b_{2}, \ldots, a_{n}+b_{n}\right) \\
\alpha u=\left(\alpha a_{1}, \alpha a_{2}, \ldots, \alpha a_{n}\right)
\end{gathered}
$$

na base $B$.

\section{Metodologia}

Nesta seção, será demonstrado que o conjunto das sequências de Fibonacci forma um espaço vetorial de dimensão 2. Dessa forma, toda sequência $f$ pode ser escrita como $f=a u+b v$, cujos coeficientes $a$ e $b$ são univocamente determinados, restando a tarefa de encontrar duas 
sequências $u$ e $v$ linearmente independentes cujos termos gerais $u_{n}$ e $v_{n}$ são funções apenas de $n$.

Definição 3.1: Dados $F_{1}, F_{2} \in \mathbb{R}$, uma sequência de Fibonacci é uma sequência $\left(f_{n}\right)_{n}=$ $\left(f_{1}, f_{2}, \ldots, f_{n}, \ldots\right)$ definida pela lei de recorrência $f_{n}=f_{n-1}+f_{n-2}, n \geq 3$. O conjunto das sequências de Fibonacci é representado por $\mathcal{F}$.

Lema 3.2: Dadas $\left(u_{n}\right)_{n},\left(v_{n}\right)_{n} \in \mathcal{F}$, então $\left(u_{n}+v_{n}\right)_{n} \in \mathcal{F}$ e $\left(\alpha u_{n}\right)_{n} \in \mathcal{F} \forall \alpha \in \mathbb{R}$.

Demonstração:

De fato, considerando as sequências $\left(s_{n}\right)_{n}=\left(u_{n}+v_{n}\right)_{n}$ e $\left(t_{n}\right)_{n}=\left(\alpha u_{n}\right)_{n}$, tem-se

$$
\begin{gathered}
s_{n}=u_{n}+v_{n}=\left(u_{n-1}+u_{n-2}\right)+\left(v_{n-1}+v_{n-2}\right)=\left(u_{n-1}+v_{n-1}\right)+\left(u_{n-2}+v_{n-2}\right)=s_{n-1}+s_{n-2} \\
t_{n}=\alpha u_{n}=\alpha\left(u_{n-1}+u_{n-2}\right)=\alpha u_{n-1}+\alpha u_{n-2}=t_{n-1}+t_{n-2}
\end{gathered}
$$

Portanto $\left(s_{n}\right)_{n}$ e $\left(t_{n}\right)_{n}$ são de Fibonacci.

Teorema 3.3: Definindo as operações $\left(u_{n}\right)_{n}+\left(v_{n}\right)_{n}=\left(u_{n}+v_{n}\right)_{n}$ e $\alpha\left(u_{n}\right)_{n}=\left(\alpha u_{n}\right), \mathcal{F}$ é um espaço vetorial com essas operações.

Demonstração: O lema 3.2 mostra que as operações de soma e produto por escalar enunciadas no teorema estão bem definidas. Para mostrar que $\mathcal{F}$ é um espaço vetorial com essas operações, as 8 condições da definição 2.1 devem estar satisfeitas. De fato, para $u=\left(u_{n}\right)_{n}, v=$ $\left(v_{n}\right)_{n}$ e $w=\left(w_{n}\right)_{n}$ pertencentes a $\mathcal{F}$ :

1. $u+v=\left(u_{n}\right)_{n}+\left(v_{n}\right)_{n}=\left(u_{n}+v_{n}\right)_{n}=\left(v_{n}+u_{n}\right)_{n}=\left(v_{n}\right)_{n}+\left(u_{n}\right)_{n}=v+u$;

2. $u+(v+w)=\left(u_{n}\right)_{n}+\left(\left(v_{n}\right)_{n}+\left(w_{n}\right)_{n}\right)=\left(u_{n}\right)_{n}+\left(v_{n}+w_{n}\right)_{n}=\left(u_{n}+\left(v_{n}+w_{n}\right)\right)_{n}=$ $\left(\left(u_{n}+v_{n}\right)+w_{n}\right)_{n}=\left(u_{n}+v_{n}\right)_{n}+\left(w_{n}\right)_{n}=\left(\left(u_{n}\right)_{n}+\left(v_{n}\right)_{n}\right)+\left(w_{n}\right)_{n}=(u+v)+w ;$

3. Basta tomar $0=(0)_{n}=(0,0, \ldots) \in \mathcal{F}$;

4. Basta tomar $-u=\left(-u_{n}\right)_{n} \in \mathcal{F}$;

5. $\alpha(\beta u)=\alpha\left(\beta\left(u_{n}\right)_{n}\right)=\alpha\left(\beta u_{n}\right)_{n}=\left(\alpha\left(\beta u_{n}\right)\right)_{n}=\left((\alpha \beta) u_{n}\right)_{n}=(\alpha \beta)\left(u_{n}\right)_{n}=(\alpha \beta) u$;

6. $\alpha(u+v)=\alpha\left(\left(u_{n}\right)_{n}+\left(v_{n}\right)_{n}\right)=\alpha\left(u_{n}+v_{n}\right)_{n}=\left(\alpha\left(u_{n}+v_{n}\right)\right)_{n}=\left(\alpha u_{n}+\alpha v_{n}\right)_{n}=$ $\left(\alpha u_{n}\right)_{n}+\left(\alpha v_{n}\right)_{n}=\alpha\left(u_{n}\right)_{n}+\alpha\left(v_{n}\right)_{n}=\alpha u+\alpha v$

7. $(\alpha+\beta) u=(\alpha+\beta)\left(u_{n}\right)_{n}=\left((\alpha+\beta) u_{n}\right)_{n}=\left(\alpha u_{n}+\beta u_{n}\right)_{n}=\left(\alpha u_{n}\right)_{n}+\left(\beta u_{n}\right)_{n}=$ $\alpha\left(u_{n}\right)_{n}+\beta\left(u_{n}\right)_{n}=\alpha u+\beta u$;

8. $1 \cdot u=1 \cdot\left(u_{n}\right)_{n}=\left(1 \cdot u_{n}\right)_{n}=\left(u_{n}\right)_{n}=u$.

Como são satisfeitas todas as condições da definição $2.1, \mathcal{F}$ é um espaço vetorial.

A seguir, será determinada a dimensão do espaço $\mathcal{F}$. Como toda sequência de Fibonacci é obtida a partir dos dois termos iniciais, é um indício de que $\operatorname{dim} \mathcal{F}=2$. De fato, isso é verdade, de acordo com o próximo teorema. 
Teorema $3.4: \operatorname{dim} \mathcal{F}=2$.

Demonstração: Procuremos dois vetores $\left(u_{n}\right)_{n}$ e $\left(v_{n}\right)_{n}$ em $\mathcal{F}$ tais que $\left\{\left(u_{n}\right)_{n},\left(v_{n}\right)_{n}\right\}$ é base de $\mathcal{F}$, isto é, que $\forall\left(f_{n}\right)_{n} \in \mathcal{F}$, existem (únicos) $a, b \in \mathbb{R}$ tais que $f_{n}=a u_{n}+b v_{n}$, o que gera o sistema

$$
\left\{\begin{array}{l}
f_{1}=a u_{1}+b v_{1} \\
f_{2}=a u_{2}+b v_{2}
\end{array}\right.
$$

Esse sistema é possível e determinado se, e somente se,

$$
\left|\begin{array}{ll}
u_{1} & v_{1} \\
u_{2} & v_{2}
\end{array}\right| \neq 0
$$

isto é, se $\left(u_{n}\right)_{n}$ e $\left(v_{n}\right)_{n}$ não são proporcionais. Tomando as sequências $(1,1,2,3,5, \ldots)$ e $(0,1,1,2,3, \ldots)$, elas são de Fibonacci e não são proporcionais, podendo assim formarem uma base para $\mathcal{F}$. Portanto $\operatorname{dim} \mathcal{F}=2$.

Resolvendo o sistema (1) encontra-se

$$
a=\frac{f_{1} v_{2}-f_{2} v_{1}}{u_{1} v_{2}-u_{2} v_{1}} \quad \text { e } \quad b=\frac{f_{2} u_{1}-f_{1} u_{2}}{u_{1} v_{2}-u_{2} v_{1}}
$$

obtendo a fórmula

$$
f_{n}=a u_{n}+b v_{n}
$$

Resta encontrar uma base $\left\{\left(u_{n}\right)_{n},\left(v_{n}\right)_{n}\right\} \subset \mathcal{F}$ onde $u_{n}$ e $v_{n}$ são funções apenas de $n$.

Observando a Figura 1, vê-se que seu gráfico se assemelha ao gráfico de uma função exponencial. Isso sugere o uso de progressões geométricas para determinar a fórmula do termo geral de uma sequência de Fibonacci, pois seus gráficos $a_{n} \times n$ são exponenciais e a fórmula do termo geral de uma progressão geométrica $\left(a_{1}, a_{2}, \ldots, a_{n}, \ldots\right)$ é (DANTE, 2009, p. 114):

$$
a_{n}=a_{1} q^{n-1}
$$

que é função somente do primeiro termo $a_{1}$ e da posição $n$.

Figura 1 - Gráfico da sequência de Fibonacci para $f_{1}=f_{2}=1$.

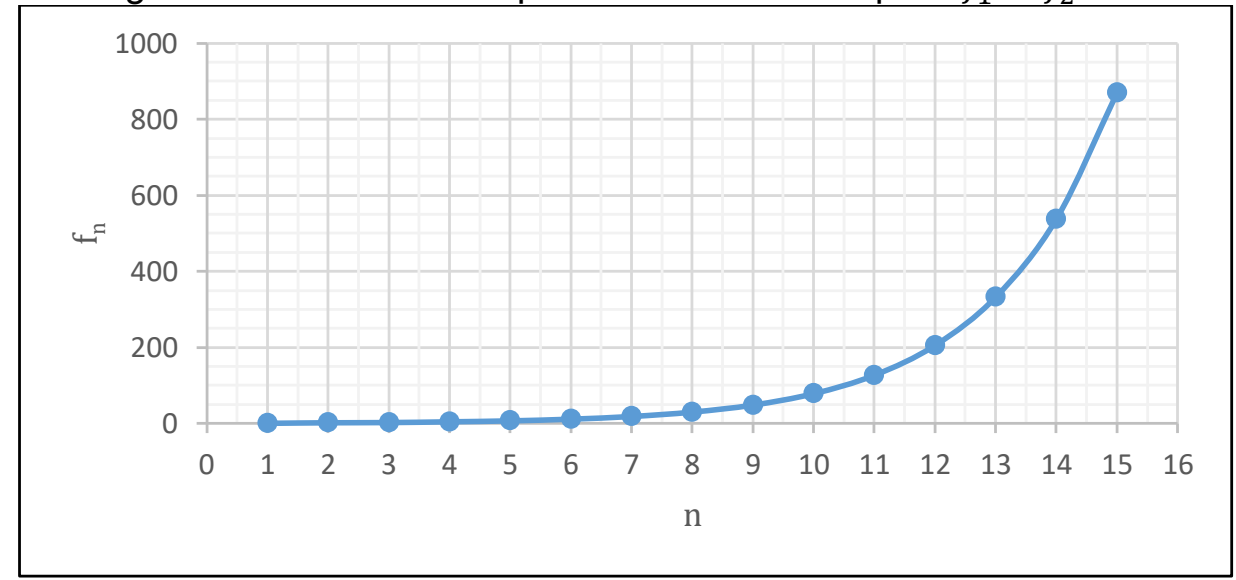

Fonte: Elaboração do autor.

Supondo que $\left(a_{n}\right)_{n}$ é de Fibonacci: 


$$
\begin{gathered}
a_{n+2}=a_{n+1}+a_{n} \Rightarrow a_{1} q^{n+1}=a_{1} q^{n}+a_{1} q^{n-1} \Rightarrow q=1+\frac{1}{q} \Rightarrow q^{2}-q-1=0 \\
\Rightarrow q=\frac{1+\sqrt{5}}{2} \quad \text { ou } \quad q=\frac{1-\sqrt{5}}{2}=1-\frac{1+\sqrt{5}}{2}
\end{gathered}
$$

O número $\frac{1+\sqrt{5}}{2}$ é chamado número de ouro e é representado pela letra grega $\phi$. As sequências $\left(\phi^{n-1}\right)_{n}$ e $\left((1-\phi)^{n-1}\right)_{n}$ são de Fibonacci, cujos termos gerais são funções apenas de $n$.

Como $\phi \neq 1-\phi,\left(\phi^{n-1}\right)_{n}$ e $\left((1-\phi)^{n-1}\right)_{n}$ não são proporcionais, são linearmente independentes e, portanto, formam uma base de $\mathcal{F}$. Com isso,

$$
f_{n}=a \phi^{n-1}+b(1-\phi)^{n-1}
$$

com

$$
a=\frac{f_{1}(1-\phi)-f_{2}}{1-2 \phi} \quad \text { e } \quad b=\frac{f_{2}-\phi f_{1}}{1-2 \phi}
$$

Substituindo em (4) e simplificando obtém-se finalmente

$$
f_{n}=\frac{\left(f_{1}+\phi f_{2}\right) \phi^{n-2}-\left(f_{2}-\phi f_{1}\right)(1-\phi)^{n-1}}{\sqrt{5}}
$$

e então está demonstrado o

Teorema 3.5: Se $\left(f_{n}\right)_{n} \in \mathcal{F}$, então

$$
f_{n}=\frac{\left(f_{1}+\phi f_{2}\right) \phi^{n-2}-\left(f_{2}-\phi f_{1}\right)(1-\phi)^{n-1}}{\sqrt{5}} .
$$

Em particular, considerando $f_{1}=f_{2}=1$, obtém-se a fórmula de Binet ${ }^{2}$

$$
f_{n}=\frac{\phi^{n}-(1-\phi)^{n}}{\sqrt{5}} \text {. }
$$

Teorema 3.6: Se $\left(f_{n}\right)_{n}$ é de Fibonacci, então

$$
\lim _{n \rightarrow \infty} \frac{f_{n+1}}{f_{n}}=\phi .
$$

Demonstração: De acordo com a Fórmula (5),

$$
\begin{aligned}
\frac{f_{n+1}}{f_{n}} & =\frac{\left(f_{1}+\phi f_{2}\right) \phi^{n-1}-\left(f_{2}-\phi f_{1}\right)(1-\phi)^{n}}{\left(f_{1}+\phi f_{2}\right) \phi^{n-2}-\left(f_{2}-\phi f_{1}\right)(1-\phi)^{n-1}} \\
& =\phi \cdot \frac{f_{1}+\phi f_{2}-\left(f_{2}-\phi f_{1}\right)\left(\frac{1}{\phi}-1\right)^{n} \frac{1}{\phi}}{f_{1}+\phi f_{2}-\left(f_{2}-\phi f_{1}\right)\left(\frac{1}{\phi}-1\right)^{n-1} \frac{1}{\phi}}
\end{aligned}
$$

\footnotetext{
${ }^{2}$ Há indícios de que tal fórmula já era conhecida por Euler e De Moivre no século XVIII, e foi redescoberta por Binet em 1843 (RAMOS, 2013, FERREIRA, 2006).
} 
Como $\left|\frac{1}{\phi}-1\right|<1$, então

$$
\lim _{n \rightarrow \infty}\left(\frac{1}{\phi}-1\right)^{n}=\lim _{n \rightarrow \infty}\left(\frac{1}{\phi}-1\right)^{n-1}=0
$$

Portanto,

$$
\lim _{n \rightarrow \infty} \frac{f_{n+1}}{f_{n}}=\phi \cdot \frac{f_{1}+\phi f_{2}}{f_{1}+\phi f_{2}}=\phi
$$

\section{Simplificação e resultados}

A seguir será feita uma análise numérica de uma versão simplificada da fórmula (5).

Como $|1-\phi|<1$, então $\lim _{n \rightarrow \infty}(1-\phi)^{n-1}=0$. Para valores suficientemente grandes de $n$ pode-se desprezar o termo $\left(f_{2}-\phi f_{1}\right)(1-\phi)^{n-1}$, obtendo

$$
f_{n} \approx \frac{f_{1}+\phi f_{2}}{\sqrt{5}} \phi^{n-2}
$$

O erro cometido é

$$
\left|\frac{\left(f_{2}-\phi f_{1}\right)(1-\phi)^{n-1}}{\sqrt{5}}\right|=\frac{\left|f_{2}-\phi f_{1}\right|}{\sqrt{5}} \phi^{1-n}
$$

Seja $\varepsilon>0$ a tolerância para o cálculo de $f_{n}$. Tem-se

$$
\frac{\left|f_{2}-\phi f_{1}\right|}{\sqrt{5}} \phi^{1-n}<\varepsilon
$$

e o índice a partir do qual o erro cometido na aplicação da fórmula (7) é inferior a $\varepsilon$ é

$$
n>1-\frac{\ln \varepsilon+0.5 \ln 5-\ln \left|f_{2}-\phi f_{1}\right|}{\ln \phi}=1-\frac{\log \varepsilon+0.5 \log 5-\log \left|f_{2}-\phi f_{1}\right|}{\log \phi}
$$

onde se pode usar logaritmos naturais ou decimais a depender da conveniência.

Por exemplo, denotando por $\llbracket x \rrbracket$ o inteiro mais próximo de $x$, na sequência original $\left(f_{n}\right)_{n}=$ $(1,1,2,3,5,8, \ldots)$, para que o erro de cálculo seja inferior a $0.5, n$ deve ser maior do que -0.23 . Isso significa que

$$
f_{n}=\llbracket \frac{\phi^{n}}{\sqrt{5}} \rrbracket
$$

para qualquer índice $n$.

Tomando, por exemplo, $f_{1}=0.5$ e $f_{2}=2$, para um erro inferior a $0.1, n$ deve ser maior do que 4.47. Ou seja, para que a fórmula (7) forneça uma aproximação em uma casa decimal para $f_{n}, n$ deve ser maior do que ou igual a 5 , como observado na Tabela 1.

O leitor pode verificar, testando outras sequências, que ocorre um ajuste entre os valores logo nos primeiros termos da sequência. Isso ocorre porque o erro absoluto decresce exponencialmente, tendendo a zero quando $n$ tende a infinito. Pode-se dizer que as fórmulas (5) e (7) se ajustam de forma assintótica. Isso explica também por que a curva da Figura 2 a seguir se estabi- 
liza nos primeiros valores de $n$ (percebe-se que após $n=7$ a curva se assemelha a uma linha reta horizontal, ou seja, ao gráfico de uma função constante).

Tabela 1 - Comparação entre as fórmulas (5) e (7) para a sequência de Fibonacci com $f_{1}=0.5$ e $f_{2}=2$.

\begin{tabular}{c|c|c|c|c}
\hline $\mathrm{n}$ & $f_{n}$ fórmula (5) & $f_{n}$ fórmula (7) & Erro absoluto & Erro relativo (\%) \\
\hline 1 & 0.50 & 1.033 & 0.533 & 106.525 \\
\hline 2 & 2.00 & 1.671 & 0.329 & 16.459 \\
\hline 3 & 2.50 & 2.703 & 0.203 & 8.138 \\
\hline 4 & 4.50 & 4.374 & 0.126 & 2.794 \\
\hline 5 & 7.00 & 7.078 & 0.078 & 1.110 \\
\hline 6 & 11.50 & 11.452 & 0.048 & 0.418 \\
\hline 7 & 18.50 & 18.530 & 0.030 & 0.160 \\
\hline 8 & 30.00 & 29.982 & 0.018 & 0.061 \\
\hline 9 & 48.50 & 48.511 & 0.011 & 0.023 \\
\hline 10 & 78.50 & 78.493 & 0.007 & 0.009 \\
\hline 11 & 127.00 & 127.004 & 0.004 & 0.003 \\
\hline 12 & 205.50 & 205.497 & 0.003 & 0.001 \\
\hline 13 & 332.50 & 332.502 & 0.002 & 0.000 \\
\hline 14 & 538.00 & 537.999 & 0.001 & 0.000 \\
\hline 15 & 870.50 & 870.501 & 0.001 & 0.000 \\
\hline \multicolumn{3}{|c|}{ Fonte: Elaboração do autor. } \\
\hline
\end{tabular}

Figura 2 - Razão $f_{n+1} / f_{n}$ para diversos valores de $n$, com $f_{1}=f_{2}=1$.

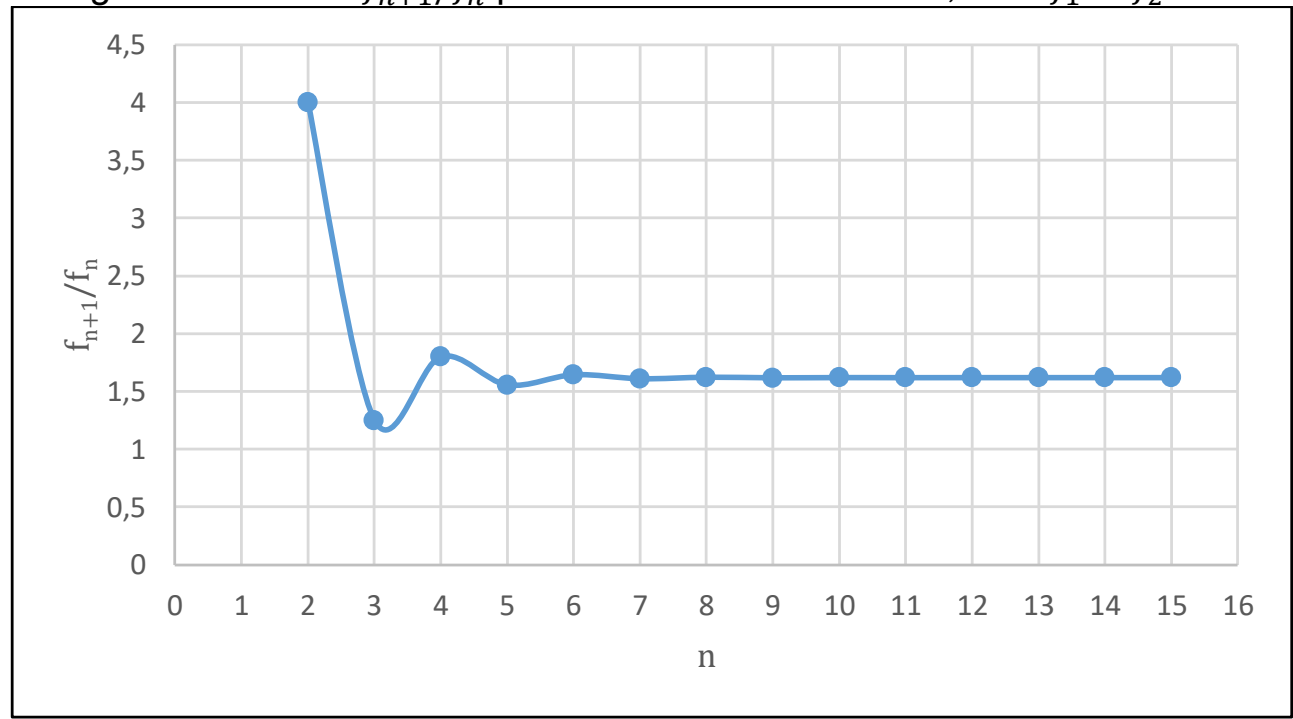

Fonte: Elaboração do autor. 
Para uma outra análise do erro cometido ao usar a fórmula (7), suponha um computador em que o menor valor em que seja possível trabalhar é $4.9 \cdot 10^{-324}$, tomando $\varepsilon=4.9 \cdot 10^{-324}$ (isto é, o menor erro possível), obtém-se

$$
n>1-\frac{-324+\log 4.9+0.5 \log 5-\log \left|f_{2}-\phi f_{1}\right|}{\log \phi} \Rightarrow n>1546.356+4.785 \log \left|f_{2}-\phi f_{1}\right|
$$

Mesmo que $\left|f_{2}-\phi f_{1}\right|$ seja relativamente grande, da ordem de $10^{6}$, por exemplo, tem-se nesse caso que $n>1575$, o que é irrelevante quando se quer trabalhar com $n$ relativamente grande, como 10000. Assim, a fórmula (7) permite uma excelente aproximação no cálculo dos termos de uma sequência de Fibonacci. Para valores pequenos de $n$, devido à presença de multiplicações, divisões, potenciações e presença de números irracionais na fórmula, é menos trabaIhoso computacionalmente fazer um cálculo recursivo.

\section{Conclusões}

Inicialmente, foi apresentado o problema que deu origem à sequência de Fibonacci $(1,1,2,3,5, \ldots)$. Em seguida, foram introduzidos conceitos básicos de Álgebra Linear como espaço vetorial, dependência linear, base e dimensão, e foram definidas sequências de Fibonacci gerais, sequências que obedecem à lei de recorrência $f_{n}=f_{n-1}+f_{n-2}, n \geq 3$. Com os conceitos apresentados na seção 2, foi possível demonstrar que o conjunto $\mathcal{F}$ das sequências de Fibonacci forma um espaço vetorial de dimensão 2. Com isso, foi obtida, com auxílio de progressões geométricas, uma base cujos termos gerais são funções apenas da posição. Mostrou-se que tal base tem relação direta com o número de ouro e, assim, obteve-se uma fórmula fechada para o termo geral de uma sequência de Fibonacci. A seguir, foi feita uma análise numérica de uma versão aproximada da fórmula, observando-se que tal permite calcular os termos de uma sequência de Fibonacci com excelente aproximação.

\section{Referências}

DANTE, L. R. Matemática: volume único. [S.I.]: Ática, 2009.

FERREIRA, R. Sequência de Fibonacci. São Paulo: UNIFIEO, 2006.

GUSMÃO, G. D. A. P. A sequência de Fibonacci. Revista da Olimpíada, v. 4, p. 55-74, jan./dez. 2003.

HUNTLEY, H. E. A divina proporção. Brasília: Universidade de Brasília, 1985.

LIMA, E. L. Álgebra linear. 9. ed. Rio de Janeiro: IMPA, 2016.

OLIVEIRA, J. J. D. Sequências de Fibonacci: possibilidades de aplicações no ensino básico. 28 f. Dissertação (Mestrado) - Mestrado Profissional em Matemática em Rede Nacional, Universidade Federal da Bahia, Salvador, 2013. 
RAMOS, M. G. O. A Sequência de Fibonacci e o Número de Ouro. 93 f. Dissertação (Mestrado) - Mestrado Profissional em Matemática em Rede Nacional, Universidade Estadual de Santa Cruz, Ilhéus, 2013.

SAMPAIO, C. F.; SILVA, A. G. D. Uma introdução à biomatemática: a importância da transdisciplinaridade entre biologia e matemática. In: VI Colóquio Internacional "Educação e Contemporaneidade", São Cristóvão: [s.n.], 2012. Disponível em:

<http://educonse.com.br/2012/eixo_06/PDF/26.pdf>. Acesso em: 22 set. 2018.

SANTOS, J. P. D. O. Introdução à teoria dos números. Rio de Janeiro: Impa, 1998.

STEINBRUCH, A.; WINTERLE, P. Introdução à álgebra linear. São Paulo: Pearson Education do Brasil, 1997. 